Journal of Accident and Emergency Medicine 1995 12, 20-22

\title{
The effect of pre-hospital administration of intravenous nalbuphine on on-scene times
}

\author{
G.S.JOHNSON \& H.R.GULY
}

Accident \& Emergency Department, Derriford Hospital, Plymouth.

\section{SUMMARY}

The use of intravenous nalbuphine in pre-hospital settings by paramedics has been demonstrated to be safe and effective. We assessed the effect of this additional intervention by comparing the time spent on-scene by ambulance crews treating patients with fractures of the tibia and fibula who received intravenous nalbuphine with those who had placement of an intravenous cannula alone and those who had neither cannula nor nalbuphine.

The mean on-scene times were $17.1 \mathrm{~min}$ without cannulation, $29.9 \mathrm{~min}$ for cannulation without nalbuphine and $37.5 \mathrm{~min}$ for cannulation and administration of nalbuphine.

The benefits of effective pre-hospital analgesia thus have a cost in terms of time. Continued audit of interventions and on-scene times is important to prevent inappropriate delays in pre-hospital care which may cause clinical and operational problems.

Key words: Analgesia, on-scene time, pre-hospital

\section{INTRODUCTION}

Since March 1992, some paramedics from the Plymouth Division of Devon Ambulance Service (now West Country Ambulance Trust) have been allowed to give nalbuphine to patients with isolated limb fractures, suspected myocardial infarction and burns. The use of intravenous nalbuphine by paramedics as a prehospital analgesic has been demonstrated to be effective and safe ${ }^{1,2}$ but requires inserting and securing an intravenous cannula, a check for contraindications to the drug followed by administration of the drug with titration of the dose against pain.

It is predictable that any increase in the level of prehospital intervention by paramedics will prolong the time spent on-scene. This may lead to clinical and operational problems. It has been demonstrated that in patients with multiple injuries, prolonged prehospital times may be associated with an increased mortality rate. ${ }^{3} \mathrm{~A}$ long on-scene time also reduces the availability of an ambulance crew to respond to other calls.

This study investigates the effect of intravenous cannulation and the administration of nalbuphine on the time spent on-scene by ambulance crews.

\section{METHODS}

Patients brought to the Accident and Emergency (A\&E) department at Derriford Hospital, Plymouth by ambulance who had a diagnosis of fracture of the shaft of the tibia and fibula in the period from the 1 May 1992 to the 31 July 1993 were identified by a search of the department's computer database. Children (less than 16 years), patients recorded as trapped at the scene and those with other injuries were excluded.

Patients were included who had been brought by both Devon Ambulance Service (some of whose paramedics were allowed to give nalbuphine) and by Cornwall Ambulance Service, which did not use nalbuphine.

Data on the insertion of an intravenous cannula, the administration of nalbuphine and the on-scene time were obtained from the pre-hospital report form filled in by the ambulance personnel. Three groups of patients were defined: those given nalbuphine, those cannulated but not given nalbuphine and those not cannulated. The mean on-scene times were calculated for each of the three groups and the differences between the groups examined by oneway analysis of variance (ANOVA).

\section{RESULTS}

A total of 106 patients over 16 years of age were identified as attending the A\&E department with a fracture of the tibia and fibula in the study period. Sixteen patients were excluded on the grounds of having other injuries. In eight cases insufficient data were available from the notes to allow analysis, leaving 82 patients in the study.

(C) 1995 Blackwell Science Ltd 
On-scene times for

pre-hospital
Number of patients Mean time on-scene (min) Range (min)

\begin{tabular}{lccc}
\hline $\begin{array}{l}\text { No cannula } \\
\text { No nalbuphine }\end{array}$ & 46 & 17.1 & $5-40$ \\
$\begin{array}{l}\text { Cannula } \\
\text { No nalbuphine }\end{array}$ & 17 & 29.9 & $16-56$ \\
$\begin{array}{l}\text { Cannula } \\
\text { Nalbuphine }\end{array}$ & 19 & 37.5 & $24-56$ \\
\hline
\end{tabular}

Table 1. Comparison of on-scene times for patients with differing on-scene interventions.
The results are shown in Table 1 . If a cannula was inserted the mean additional on-scene time was $\mathbf{1 2 . 8}$ minutes and if nalbuphine is administered the mean additional time was a further $7.6 \mathrm{~min}$. One-way analysis of variance demonstrated a significant difference between the three groups $(P<$ 0.001).

\section{DISCUSSION}

The additional time of $7.6 \mathrm{~min}$ to give nalbuphine in a cannulated patient is not excessive considering that the protocol was to titrate the drug at $4 \mathrm{mg}$ $\min ^{-1}$. The mean time to cannulation seems excessive but is similar to those found by others ${ }^{4}$ and is almost identical to that found in a previous study of Cornwall Ambulance Service ${ }^{5}$ (one of the ambulance services involved in this study). However it has been demonstrated that with good training and good medical control, insertion times for intravenous cannulae can be as low as 2.2 min. $^{6}$

It is possible that patients who were given nalbuphine or who were cannulated had more severe injuries than patients who had no advanced skills applied and this might account for some of the additional on-scene time but this is unlikely as many ambulance personnel were not qualified to give nalbuphine. Rouse ${ }^{5}$ has previously demonstrated that the decision to start an intravenous infusion seems to depend on the skills of the ambulance crew rather than the clinical need.

Trunkey ${ }^{7}$ has identified the following three areas of major controversy in pre-hospital care.

(1) Which procedures are useful in the field?

(2) What is the balance between time spent in the field and clinical benefit?

(3) Medical control of prehospital care.

In this example of the use of intravenous nalbuphine in patients with isolated limb fractures, the benefit in terms of improved analgesia is weighed crews and therefore reduced availability of the ambulances. It is unlikely that the delay in patients with an isolated limb fracture arriving at hospital was clinically detrimental.

As the number and complexity of pre-hospital interventions increase, more time can be spent by ambulance crews treating patients on-scene. It is important to assess the effect of pre-hospital interventions to ensure that they produce benefits to the patient without excessive delay in definitive care. When on-scene times are found to be long, continuing audit of practice can produce significant reductions. $^{8}$

In the United States it has been found that medical direction of the Emergency Medical Services is necessary to ensure satisfactory standards of paramedical care and that medical input from the Emergency Department is required to ensure that skills are used appropriately at the scene and are not causing excessive delays. ${ }^{3}$ If similar problems are to be avoided in this country, especially as more ambulance services gain increased autonomy as trusts, a mechanism must be available to allow medical input in the determination of appropriateness of pre-hospital care.

\section{REFERENCES}

1. Chambers J. \& Guly H.R. (1994) Prehospital intravenous nalbuphine administered by paramedics. Resuscitation 27, 153-158.

2. Stene J.R., Stofberg L., MacDonald G., Myers R.A., Ramzy A. \& Burns B. (1988) Nalbuphine analgesia in the prehospital setting. American Journal of Emergency Medicine 6, 634-639.

3. Border J.R., Lewis F.R., Aprahamian C., Haller J.A., Jacobs L.M. \& Luterman A. (1983) Prehospital trauma care - stabilize or scoop and run. Journal of Trauma 23, 708-711.

4. McSwain N.E., Garrison W.B., Artz C.P. (1985) Evaluation of resuscitation from cardiopulmonary arrest by paramedics. Journal of Trauma 9, 341-345. 
G.S. Johnson \&

H.R. Guly
5. Rouse A. (1991) Do ambulance crews triage patients? Archives of Emergency Medicine 8, 185-91.

6. Pons P.T., Moore E.E., Cusick J.M., Brunko M., Antuna B. \& Owens L. (1988) Prehospital venous access in an urban paramedic system. Journal of Trauma 28, 1460-1463.
7. Trunkey D.D. (1984) Is ALS necessary for prehospital trauma care? Journal of Trauma 24, 86-87.

8. Werman H.A., Nelson R.N. \& Campbell J.E. (1987) Basic Trauma Life Support. Annals of Emergency Medicine 16, 1240-1243. 